\title{
Influence of dietary insulin scores on survival in colorectal cancer patients
}

Chen Yuan ${ }^{\star}, 1,2$, Ying Bao ${ }^{3}$, Kaori Sato ${ }^{1}$, Katharina Nimptsch ${ }^{4,5}$, Mingyang Song ${ }^{4,6}$, Jennie C Brand-Miller ${ }^{7}$, Vicente Morales-Oyarvide ${ }^{1}$, Emilie S Zoltick ${ }^{2}$, NaNa Keum ${ }^{4,8}$, Brian M Wolpin ${ }^{1}$, Jeffrey A Meyerhardt ${ }^{1}$, Andrew T Chan ${ }^{6}$, Walter C Willett ${ }^{2,3,4}$, Meir J Stampfer ${ }^{2,3,4}$, Kana Wu ${ }^{4}$, Edward L Giovannucci ${ }^{2,3,4}$, Charles S Fuchs ${ }^{9}$ and Kimmie $\mathrm{Ng}^{1}$

${ }^{1}$ Department of Medical Oncology, Dana-Farber Cancer Institute and Harvard Medical School, 450 Brookline Avenue, Boston, MA 02215, USA; ${ }^{2}$ Department of Epidemiology, Harvard T.H. Chan School of Public Health, Boston, MA, USA; ${ }^{3}$ Channing Division of Network Medicine, Department of Medicine, Brigham and Women's Hospital and Harvard Medical School, Boston, MA, USA; ${ }^{4}$ Department of Nutrition, Harvard T.H. Chan School of Public Health, Boston, MA, USA; ${ }^{5}$ Molecular Epidemiology Research Group, Max Delbrück Center for Molecular Medicine (MDC), Berlin, Germany; ${ }^{6}$ Clinical and Translational Epidemiology Unit and Division of Gastroenterology, Massachusetts General Hospital and Harvard Medical School, Boston, MA, USA; ${ }^{7}$ Institute of Obesity, Nutrition, and Exercise, University of Sydney, Sydney, Australia; ${ }^{8}$ Department of Food Science and Biotechnology, Dongguk University, Goyang, South Korea and ${ }^{9}$ Yale Cancer Center, New Haven, CT, USA

Background: Although hyperinsulinemia is hypothesised to be involved in colorectal carcinogenesis, it remains unclear whether a diet inducing an elevated insulin response influences colorectal cancer (CRC) survival.

Methods: We examined the association of post-diagnosis dietary insulin scores with survival among 2006 patients from two large prospective cohorts who were diagnosed with CRC from 1976 to 2010. Dietary insulin load was calculated as a function of the food insulin index. Dietary insulin index was calculated by dividing insulin load by total energy intake. Cox proportional hazards models were used to calculate hazard ratios (HRs) for CRC-specific mortality and overall mortality, adjusted for other risk factors for cancer survival.

Results: The adjusted HRs for CRC-specific mortality comparing the highest to the lowest quintiles were 1.82 (95\% Cl: 1.20-2.75, $\left.P_{\text {trend }}=0.006\right)$ for dietary insulin load and $1.66\left(95 \% \mathrm{Cl}: 1.10-2.50, P_{\text {trend }}=0.004\right)$ for dietary insulin index. We also observed an increased risk for overall mortality, with adjusted HRs of $1.33\left(95 \% \mathrm{Cl}: 1.03-1.72, P_{\text {trend }}=0.03\right)$ for dietary insulin load and $1.32(95 \%$ $\left.\mathrm{Cl}: 1.02-1.71, P_{\text {trend }}=0.02\right)$ for dietary insulin index, comparing extreme quintiles. The increase in CRC-specific mortality associated with higher dietary insulin scores was more apparent among patients with body mass index (BMI) $\geqslant 25 \mathrm{~kg} \mathrm{~m}^{-2}$ than $\mathrm{BMl}<25 \mathrm{~kg} \mathrm{~m}^{-2}\left(P_{\text {interaction }}=0.01\right)$.

Conclusions: Higher dietary insulin scores after CRC diagnosis were associated with a statistically significant increase in CRCspecific and overall mortality.

Hyperinsulinemia and insulin resistance are hypothesised to play important roles in the development of colorectal cancer (CRC). Many of the established risk factors for CRC, including obesity (Giovannucci, 2003a; Moghaddam et al, 2007), sedentary lifestyle (Samad et al, 2005) and type 2 diabetes mellitus (Larsson et al,
2005), are characterised by hyperinsulinemia and insulin resistance. Higher circulating insulin and C-peptide (a marker of insulin resistance and long-term insulin secretion) have also been associated with an increased risk of CRC in many studies (Schoen et al, 1999; Kaaks et al, 2000; Ma et al, 2004; Wei et al, 2005).

*Correspondence: Dr C Yuan; E-mail: chen_yuan@dfci.harvard.edu

Received 28 March 2017; revised 27 June 2017; accepted 21 July 2017; published online 17 August 2017

(C) 2017 Cancer Research UK. All rights reserved 0007-0920/17 
Beyond cancer risk, increasing evidence indicates that the same host factors, including obesity (Meyerhardt et al, 2003), sedentary lifestyle (Meyerhardt et al, 2006a), and high intake of a Western pattern diet (Meyerhardt et al, 2007) are associated with an increased risk of recurrence or death among CRC patients.

The association between hyperinsulinemia and CRC suggests that a diet inducing an elevated insulin response may contribute to tumour growth. Dietary glycaemic load and dietary glycaemic index have been used to quantify the influence of carbohydrate intake on blood glucose. A recent study showed that higher dietary glycaemic load, but not dietary glycaemic index, was associated with an increased risk of recurrence and death in stage III colon cancer patients (Meyerhardt et al, 2012). However, dietary glycaemic scores, which only reflect carbohydrate intake, may be suboptimal indicators of insulin response since protein and fat intake can also increase insulin secretion.

A novel insulin score was therefore developed to quantify postprandial insulin response for various food items, including those with low or no carbohydrate content (Holt et al, 1997). Using this new measure, the insulin response to overall diets, represented by dietary insulin load and dietary insulin index, can be calculated. In a validation study, dietary insulin index was strongly correlated with actual circulating insulin concentrations ( $r=0.78, P=0.0016)$, and led to a more accurate prediction of insulin demand evoked by composite meals than carbohydrate content or dietary glycaemic load (Bao et al, 2009).

In this study, we used these two dietary insulin scores to investigate whether diets high in foods that increase postprandial insulin concentrations influence survival among CRC patients from two large prospective cohort studies, the Nurses' Health Study (NHS) and the Health Professionals Follow-Up Study (HPFS). We also examined the association of dietary insulin scores with CRC survival across strata of relevant biomarkers, including adiponectin (Chong et al, 2015), C-peptide (Wolpin et al, 2009), and insulin-like growth factor binding protein (IGFBP)-1 (Wolpin et al, 2009), among a subset of patients with plasma samples collected before cancer diagnosis.

\section{MATERIALS AND METHODS}

Study population. NHS was initiated in 1976 when 121700 U.S. female nurses aged 30-55 years completed a mailed questionnaire describing demographics, lifestyle choices, and medical history (Colditz et al, 1997). HPFS was initiated in 1986 when 51529 U.S. men aged 40-75 years working in health professions completed a mailed questionnaire on health-related behaviours and medical history (Rimm et al, 1991). Since then, participants have updated information through biennial follow-up questionnaires. All participants were enrolled at baseline. This study was approved by the Human Subjects Committee at the Brigham and Women's Hospital and Harvard T.H. Chan School of Public Health, and all participants provided informed consent.

Identification of study patients. NHS and HPFS participants with pathologically confirmed colorectal adenocarcinoma were identified after return of the baseline questionnaire (NHS: 1976; HPFS: 1986) through 2010. When a participant (or next of kin for decedents) reported a diagnosis of CRC during the previous two years on follow-up questionnaires, we asked permission to obtain hospital records and pathology reports. Blinded study physicians then reviewed these records and recorded information on important tumour characteristics. For nonrespondents, the National Death Index was used to discover deaths and ascertain any diagnosis of CRC that contributed to death or was a secondary diagnosis. We estimate that $96-97 \%$ of patients were identified through these methods (Giovannucci et al, 1994a,b). We excluded participants who had reported any cancer (other than nonmelanoma skin cancer) before CRC diagnosis, who had diabetes at CRC diagnosis (because diabetic patients usually limit intake of insulinogenic foods), and who died within three months of dietary assessment (to minimise bias by occult recurrence or impeding death).

Mortality assessment. Ascertainment of deaths included reporting by family or postal authorities, and interrogation of names of persistent nonresponders in the National Death Index (Sathiakumar et al, 1998). More than 98\% of deaths have been identified by these methods (Stampfer et al, 1984). Cause of death was assigned by blinded physicians.

Dietary assessment. Dietary intake was obtained from NHS participants via validated semiquantitative food-frequency questionnaires (FFQs) in 1980, 1984, 1986, and every 4 years thereafter, and from HPFS participants every 4 years starting in 1986. Participants were asked to report their average frequency of intake over the preceding year for a specified serving size of each food. Individual nutrient intakes were calculated by multiplying the frequency of each food consumed by the nutrient content of the specified portion size, and then summing the contributions from all foods.

The insulin index value for each food item compares the postprandial plasma insulin response induced by that food relative to that of a reference food (glucose or white bread). Insulin index values for foods that appeared in the FFQ were obtained either from published estimates (31 foods) (Holt et al, 1997) or from direct testing of food items at the University of Sydney, Australia (73 foods; provided by Jennie Brand-Miller). U.S. food samples were shipped to the laboratory in Sydney for testing. The testing procedure has been described in detail previously (Bao et al, 2009): each person consumed a variety of test foods on separate days, with insulin measured every $15 \mathrm{~min}$ for $2 \mathrm{~h}$ after consumption. The food insulin index value was calculated by dividing the area under the insulin response curve for $1000 \mathrm{~kJ}$ of a test food by the area under the insulin response curve for $1000 \mathrm{~kJ}$ of the reference food. The insulin index value for each food represented the mean responses of 11-13 participants.

Using these insulin index values, we calculated the average dietary insulin load for each participant by multiplying the insulin index value of each food by the total energy intake contributed by that food, and summing values for all food items reported:

Dietary insulin load

$$
=\sum\left[\begin{array}{l}
\text { food insulin index } \times \text { energy content of food } \\
(\mathrm{kcal} / \text { serving }) \times \text { frequency of consumption } \\
(\text { servings/day })
\end{array}\right]
$$

Each unit represents the equivalent insulin response generated by 1 kilocalorie of the reference food. The dietary insulin index for the overall diet, which is the weighted mean of the insulin index values for each of the component foods, was calculated by dividing insulin load by total energy intake:

$$
\begin{aligned}
& \text { Dietary insulin index } \\
& =\text { dietary insulin load } / \sum\left[\begin{array}{l}
\text { energy content of food }(\mathrm{kcal} / \text { serving }) \\
\times \text { frequency of consumption } \\
(\text { servings/day })
\end{array}\right]
\end{aligned}
$$

Dietary insulin load and dietary insulin index were energyadjusted by the residual method (Willett and Stampfer, 1986). The FFQ was found to be a reasonably accurate measure of a person's food intake (Salvini et al, 1989; Feskanich et al, 1993). For top food sources contributing to dietary insulin load, the Pearson 
Table 1. Baseline characteristics among patients with colorectal cancer by quintile of post-diagnosis dietary insulin score

\begin{tabular}{|c|c|c|c|c|c|c|c|c|c|c|}
\hline \multirow[b]{2}{*}{ Characteristic } & \multicolumn{5}{|c|}{ Dietary insulin load } & \multicolumn{5}{|c|}{ Dietary insulin index } \\
\hline & Quintile 1 & Quintile 2 & Quintile 3 & Quintile 4 & Quintile 5 & Quintile 1 & Quintile 2 & Quintile 3 & Quintile 4 & Quintile 5 \\
\hline No. of patients & 401 & 404 & 400 & 402 & 399 & 400 & 401 & 402 & 402 & 401 \\
\hline $\begin{array}{l}\text { Age at diagnosis, years, mean } \\
\text { (s.d.) }\end{array}$ & $66.9(9.9)$ & $67.1(9.7)$ & $66.4(10.0)$ & $66.7(9.2)$ & $68.8(8.7)$ & $66.7(10.3)$ & $67.0(9.5)$ & $66.2(10.1)$ & $67.5(8.9)$ & $68.6(8.8)$ \\
\hline $\begin{array}{l}\text { Sex, No. (\%) } \\
\text { Female } \\
\text { Male }\end{array}$ & $\begin{array}{l}262(65.3) \\
139(34.7)\end{array}$ & $\begin{array}{l}266(65.8) \\
138(34.2)\end{array}$ & $\begin{array}{l}262(65.5) \\
138(34.5)\end{array}$ & $\begin{array}{l}264(65.7) \\
138(34.3)\end{array}$ & $\begin{array}{l}260(65.2) \\
139(34.8)\end{array}$ & $\begin{array}{l}261(65.3) \\
139(34.8)\end{array}$ & $\begin{array}{l}264(65.8) \\
137(34.2)\end{array}$ & $\begin{array}{l}263(65.4) \\
139(34.6)\end{array}$ & $\begin{array}{l}263(65.4) \\
139(34.6)\end{array}$ & $\begin{array}{l}263(65.6) \\
138(34.4)\end{array}$ \\
\hline $\begin{array}{l}\text { Race, No. (\%) } \\
\text { White } \\
\text { Black } \\
\text { Other } \\
\text { Unknown }\end{array}$ & $\begin{aligned} 385 & (96.0) \\
4 & (1.0) \\
2 & (0.5) \\
10 & (2.5)\end{aligned}$ & $\begin{array}{c}392(97.0) \\
3(0.7) \\
4(1.0) \\
5(1.2)\end{array}$ & $\begin{array}{c}382(95.5) \\
\quad 9(2.3) \\
6(1.5) \\
3(0.8)\end{array}$ & $\begin{array}{c}385(95.8) \\
\quad 4(1.0) \\
4(1.0) \\
9(2.2)\end{array}$ & $\begin{aligned} 381 & (95.5) \\
3 & (0.8) \\
7 & (1.8) \\
8 & (2.0)\end{aligned}$ & $\begin{array}{c}384(96.0) \\
4(1.0) \\
2(0.5) \\
10(2.5)\end{array}$ & $\begin{array}{c}389(97.0) \\
3(0.7) \\
4(1.0) \\
5(1.2)\end{array}$ & $\begin{aligned} 385 & (95.8) \\
7 & (1.7) \\
7 & (1.7) \\
3 & (0.7)\end{aligned}$ & $\begin{array}{c}386(96.0) \\
5(1.2) \\
3(0.7) \\
8(2.0)\end{array}$ & $\begin{aligned} 381 & (95.0) \\
\quad 4 & (1.0) \\
7 & (1.7) \\
9 & (2.2)\end{aligned}$ \\
\hline Current smoker, No. (\%) & $47(11.7)$ & $38(9.4)$ & $23(5.8)$ & $18(4.5)$ & $15(3.8)$ & $47(11.8)$ & $35(8.7)$ & $25(6.2)$ & $18(4.5)$ & $16(4.0)$ \\
\hline $\begin{array}{l}\text { Body mass index, } \mathrm{kg} \mathrm{m}^{-2} \text {, } \\
\text { mean (s.d.) }\end{array}$ & $25.8(4.3)$ & $25.6(4.5)$ & $25.8(4.7)$ & $25.8(4.5)$ & $25.5(4.6)$ & $25.9(4.3)$ & $25.7(4.5)$ & $25.7(4.8)$ & $25.8(4.5)$ & $25.4(4.5)$ \\
\hline $\begin{array}{l}\text { Physical activity, MET-h per } \\
\text { week, median }(\text { range) }\end{array}$ & $11.2(0-221.9)$ & $10.1(0-145.9)$ & $10.9(0-172.0)$ & $9.6(0-168.4)$ & $9.8(0-125.0)$ & $11.2(0-221.9)$ & $10.2(0-145.9)$ & $10.4(0-172.0)$ & $10.1(0-168.4)$ & $9.7(0-125.0)$ \\
\hline $\begin{array}{l}\text { Alcohol intake, } \mathrm{gd}^{-1}, \text { median } \\
\text { (range) }\end{array}$ & $14.0(0-88.5)$ & $3.1(0-76.8)$ & $1.8(0-65.0)$ & $0.9(0-41.4)$ & $0(0-47.0)$ & $14.3(0-88.5)$ & $3.3(0-63.9)$ & $1.8(0-65.0)$ & $0.9(0-41.4)$ & $0(0-47.0)$ \\
\hline $\begin{array}{l}\text { Vitamin } \mathrm{D} \text { intake, } \mathrm{IUd}^{-1} \text {, } \\
\text { energy-adjusted, median } \\
\text { (range) }\end{array}$ & $488(15-3179)$ & $400(29-2955)$ & $444(14-2635)$ & $425(29-2543)$ & $523(21-2436)$ & $490(15-3179)$ & 398 (14-2955) & $416(29-2635)$ & $446(29-2543)$ & $517(21-2436)$ \\
\hline $\begin{array}{l}\text { Carbohydrate intake, } \mathrm{gd}^{-1} \text {, } \\
\text { energy-adjusted, median } \\
\text { (range) }\end{array}$ & $182(49-317)$ & $206(116-339)$ & $222(143-352)$ & $232(146-335)$ & 259 (194-394) & $181(49-317)$ & $204(111-349)$ & $222(143-352)$ & $232(146-348)$ & $258(172-394)$ \\
\hline $\begin{array}{l}\text { Protein intake, } \mathrm{gd}^{-1} \text {, energy- } \\
\text { adjusted, median (range) }\end{array}$ & $74(36-141)$ & $76(31-135)$ & $74(35-129)$ & $73(41-133)$ & $70(27-129)$ & $74(36-141)$ & $75(43-135)$ & 74 (31-129) & $72(43-133)$ & $69(27-129)$ \\
\hline $\begin{array}{l}\text { Total fat intake, } \mathrm{gd}^{-1} \text {, energy- } \\
\text { adjusted, median (range) }\end{array}$ & $66(24-140)$ & $63(18-108)$ & 59 (31-98) & 55 (26-94) & $47(23-85)$ & $66(24-140)$ & $63(18-108)$ & $59(31-98)$ & $56(26-94)$ & $47(23-85)$ \\
\hline $\begin{array}{l}\text { Energy intake, kcal d }{ }^{-1} \text {, } \\
\text { median (range) }\end{array}$ & $1716(601-4004)$ & 1705 (660-3993) & $1749(627-3838)$ & $1729(611-3761)$ & $1655(611-4194)$ & $1713(601-4004)$ & $1722(660-3993)$ & $1747(627-3838)$ & $1722(628-3770)$ & $1649(611-4194)$ \\
\hline Stage, No. (\%) & & & & & & & & & & \\
\hline $\begin{array}{l}\text { I } \\
\text { II } \\
\text { III } \\
\text { IV } \\
\text { Unknown }\end{array}$ & $\begin{array}{c}142(35.4) \\
111(27.7) \\
85(21.2) \\
22(5.5) \\
41(10.2)\end{array}$ & $\begin{array}{c}134(33.2) \\
103(25.5) \\
92(22.8) \\
28(6.9) \\
47(11.6)\end{array}$ & $\begin{array}{c}109(27.3) \\
118(29.5) \\
103(25.8) \\
22(5.5) \\
48(12.0)\end{array}$ & $\begin{array}{c}132(32.8) \\
122(30.3) \\
84(20.9) \\
22(5.5) \\
42(10.4)\end{array}$ & $\begin{array}{c}119(29.8) \\
114(28.6) \\
91(22.8) \\
24(6.0) \\
51(12.8)\end{array}$ & $\begin{array}{c}141(35.3) \\
109(27.3) \\
89(22.3) \\
22(5.5) \\
39(9.8)\end{array}$ & $\begin{array}{c}139(34.7) \\
103(25.7) \\
85(21.2) \\
27(6.7) \\
47(11.7)\end{array}$ & $\begin{array}{c}111(27.6) \\
116(28.9) \\
103(25.6) \\
24(6.0) \\
48(11.9)\end{array}$ & $\begin{array}{c}126(31.3) \\
128(31.8) \\
83(20.6) \\
23(5.7) \\
42(10.4)\end{array}$ & $\begin{array}{c}119(29.7) \\
112(27.9) \\
95(23.7) \\
22(5.5) \\
53(13.2)\end{array}$ \\
\hline $\begin{array}{l}\text { Grade of tumour } \\
\text { differentiation, No. (\%) }\end{array}$ & & & & & & & & & & \\
\hline $\begin{array}{l}\text { Well } \\
\text { Moderate } \\
\text { Poor } \\
\text { Unknown }\end{array}$ & $\begin{array}{l}167(41.6) \\
133(33.2) \\
81(20.2) \\
20(5.0)\end{array}$ & $\begin{array}{c}175(43.3) \\
114(28.2) \\
97(24.0) \\
18(4.5)\end{array}$ & $\begin{array}{c}154(38.5) \\
130(32.5) \\
94(23.5) \\
22(5.5)\end{array}$ & $\begin{array}{c}166(41.3) \\
130(32.3) \\
88(21.9) \\
18(4.5)\end{array}$ & $\begin{array}{l}169(42.4) \\
129(32.3) \\
84(21.1) \\
17(4.3)\end{array}$ & $\begin{array}{r}166(41.5) \\
130(32.5) \\
84(21.0) \\
20(5.0)\end{array}$ & $\begin{array}{c}168(41.9) \\
117(29.2) \\
98(24.4) \\
18(4.5)\end{array}$ & $\begin{array}{c}153(38.1) \\
140(34.8) \\
87(21.6) \\
22(5.5)\end{array}$ & $\begin{array}{c}177(44.0) \\
116(28.9) \\
92(22.9) \\
17(4.2)\end{array}$ & $\begin{array}{c}167(41.6) \\
133(33.2) \\
83(20.7) \\
18(4.5)\end{array}$ \\
\hline $\begin{array}{l}\text { Location of primary tumour, } \\
\text { No. (\%) }\end{array}$ & & & & & & & & & & \\
\hline Proximal colon & $70(17.5)$ & $57(14.1)$ & $53(13.3)$ & $67(16.7)$ & $44(11.0)$ & $70(17.5)$ & $60(15.0)$ & $45(11.2)$ & $71(17.7)$ & $45(11.2)$ \\
\hline Distal colon & $213(53.1)$ & $218(54.0)$ & $235(58.8)$ & $216(53.7)$ & $243(60.9)$ & $213(53.3)$ & $215(53.6)$ & $236(58.7)$ & $226(56.2)$ & $235(58.6)$ \\
\hline Rectum & $54(13.5)$ & $59(14.6)$ & $50(12.5)$ & $55(13.7)$ & $47(11.8)$ & $53(13.3)$ & $53(13.2)$ & $58(14.4)$ & $52(12.9)$ & $49(12.2)$ \\
\hline Unknown & $64(16.0)$ & $70(17.3)$ & $62(15.5)$ & $64(15.9)$ & $65(16.3)$ & $64(16.0)$ & $73(18.2)$ & $63(15.7)$ & $53(13.2)$ & $72(18.0)$ \\
\hline
\end{tabular}

correlation coefficients between FFQ and one-week diet records were listed in Supplementary Table 1.

Covariates. Stage of disease, grade of tumour differentiation, primary tumour location, and year of diagnosis (as a surrogate for treatment) were extracted from the medical record. Body mass index, physical activity, and smoking status were taken from the same questionnaire that assessed dietary insulin scores.

Measurement of biomarkers. In NHS, a total of 32826 women between 43 and 69 years of age returned a mailed blood collection kit by overnight courier in 1989 and 1990. In HPFS, blood was collected from 18225 men and returned in a mailed blood collection kit by overnight courier in 1993 through 1995. Approximately $95 \%$ of samples were received within $24 \mathrm{~h}$ of blood collection.
Plasma levels of adiponectin were measured using ELISA from ALPCO Diagnostics. Plasma levels of C-peptide and IGFBP-1 were measured by enzyme-linked immunosorbent assays with reagents from Diagnostic Systems Laboratory (Webster, TX, USA) in the laboratory of Michael Pollak (McGill University, Montreal, Canada). The mean intra-assay coefficients of variation for these biomarkers were all $<13 \%$.

Statistical analyses. The primary exposure was post-diagnosis dietary insulin load and dietary insulin index, calculated from the first dietary assessment within 4 years of diagnosis (median, 21 months). We categorised the dietary insulin score into quintiles, with cutoffs determined separately within NHS and HPFS, and combined the cohorts for pooled analysis. Follow-up time was calculated from post-diagnosis dietary assessment to death or last follow-up dates, June 2012 in NHS, or January 2012 in HPFS, 
Table 2. HRs for CRC-specific and overall mortality among patients with colorectal cancer by quintile of post-diagnosis dietary insulin score

\begin{tabular}{|c|c|c|c|c|c|c|}
\hline Dietary insulin score & Quintile 1 & Quintile 2 & Quintile 3 & Quintile 4 & Quintile 5 & $P_{\text {trend }}$ \\
\hline \multicolumn{7}{|l|}{ Dietary insulin load } \\
\hline $\begin{array}{l}\text { Median (range) } \\
\text { Nurses' Health Study } \\
\text { Health Professionals Follow-up Study }\end{array}$ & $\begin{array}{l}582(298-625) \\
693(428-748)\end{array}$ & $\begin{array}{l}652(626-674) \\
783(749-810)\end{array}$ & $\begin{array}{l}697(675-714) \\
837(811-862)\end{array}$ & $\begin{array}{l}736(715-763) \\
888(863-923)\end{array}$ & $\begin{array}{l}804(765-1104) \\
967(924-1258)\end{array}$ & \\
\hline $\begin{array}{l}\text { CRC-specific mortality } \\
\text { Events/patients } \\
\text { Age-adjusted HR }(95 \% \mathrm{Cl}) \\
\text { Multivariable-adjusted HR }(95 \% \mathrm{Cl})^{\mathrm{a}}\end{array}$ & $\begin{array}{l}47 / 401 \\
\text { Referent } \\
\text { Referent }\end{array}$ & $\begin{array}{c}69 / 404 \\
1.44(0.99-2.08) \\
1.31(0.89-1.94)\end{array}$ & $\begin{array}{c}77 / 400 \\
1.62(1.13-2.33) \\
1.55(1.04-2.29)\end{array}$ & $\begin{array}{c}72 / 402 \\
1.42(0.99-2.06) \\
1.48(0.99-2.21)\end{array}$ & $\begin{array}{c}78 / 399 \\
1.70(1.18-2.44) \\
1.82(1.20-2.75)\end{array}$ & $\begin{array}{l}0.01 \\
0.006\end{array}$ \\
\hline $\begin{array}{l}\text { Overall mortality } \\
\text { Events/patients } \\
\text { Age-adjusted HR }(95 \% \mathrm{Cl}) \\
\text { Multivariable-adjusted HR }(95 \% \mathrm{Cl})^{\mathbf{a}}\end{array}$ & $\begin{array}{l}145 / 401 \\
\text { Referent } \\
\text { Referent }\end{array}$ & $\begin{array}{c}166 / 404 \\
1.09(0.87-1.37) \\
1.13(0.89-1.43)\end{array}$ & $\begin{array}{c}157 / 400 \\
1.02(0.81-1.28) \\
1.11(0.87-1.41)\end{array}$ & $\begin{array}{c}171 / 402 \\
1.02(0.82-1.28) \\
1.17(0.92-1.50)\end{array}$ & $\begin{array}{c}176 / 399 \\
1.11(0.89-1.39) \\
1.33(1.03-1.72)\end{array}$ & $\begin{array}{l}0.48 \\
0.03\end{array}$ \\
\hline \multicolumn{7}{|l|}{ Dietary insulin index } \\
\hline $\begin{array}{l}\text { Median (range) } \\
\text { Nurses' Health Study } \\
\text { Health Professionals Follow-up Study }\end{array}$ & $\begin{array}{l}36(20-39) \\
35(21-37)\end{array}$ & $\begin{array}{l}41(39-42) \\
39(37-40)\end{array}$ & $\begin{array}{l}44(42-45) \\
42(41-43)\end{array}$ & $\begin{array}{l}46(45-48) \\
44(43-46)\end{array}$ & $\begin{array}{l}50(48-70) \\
48(46-63)\end{array}$ & \\
\hline $\begin{array}{l}\text { CRC-specific mortality } \\
\text { Events/patients } \\
\text { Age-adjusted HR }(95 \% \mathrm{Cl}) \\
\text { Multivariable-adjusted HR }(95 \% \mathrm{Cl})^{\mathrm{a}}\end{array}$ & $\begin{array}{l}50 / 400 \\
\text { Referent } \\
\text { Referent }\end{array}$ & $\begin{array}{c}62 / 401 \\
1.21(0.84-1.76) \\
1.17(0.79-1.73)\end{array}$ & $\begin{array}{c}74 / 402 \\
1.42(0.99-2.04) \\
1.32(0.89-1.95)\end{array}$ & $\begin{array}{c}82 / 402 \\
1.58(1.11-2.25) \\
1.60(1.09-2.36)\end{array}$ & $\begin{array}{c}75 / 401 \\
1.51(1.05-2.16) \\
1.66(1.10-2.50)\end{array}$ & $\begin{array}{l}0.008 \\
0.004\end{array}$ \\
\hline $\begin{array}{l}\text { Overall mortality } \\
\text { Events/patients } \\
\text { Age-adjusted HR }(95 \% \mathrm{Cl}) \\
\text { Multivariable-adjusted HR }(95 \% \mathrm{Cl})^{\mathrm{a}}\end{array}$ & $\begin{array}{l}142 / 400 \\
\text { Referent } \\
\text { Referent }\end{array}$ & $\begin{array}{c}160 / 401 \\
1.09(0.87-1.36) \\
1.17(0.92-1.49)\end{array}$ & $\begin{array}{c}156 / 402 \\
1.00(0.79-1.25) \\
1.07(0.83-1.37)\end{array}$ & $\begin{array}{c}186 / 402 \\
1.17(0.94-1.46) \\
1.35(1.06-1.72)\end{array}$ & $\begin{array}{c}171 / 401 \\
1.08(0.86-1.35) \\
1.32(1.02-1.71)\end{array}$ & $\begin{array}{l}0.39 \\
0.02\end{array}$ \\
\hline $\begin{array}{l}\text { Abbreviations: } \mathrm{Cl}=\text { confidence interval; } \mathrm{CRC}= \\
\text { a Adjusted for age at diagnosis (continuous), se } \\
\geqslant 35.0 \mathrm{~kg} \mathrm{~m}^{-2} \text {, or unknown), physical activity (c } \\
\text { moderate, poor, unknown), location of primary }\end{array}$ & $\begin{array}{l}\text { tal cancer; } \mathrm{HR}= \\
\text { White, Black, ot } \\
\text { or unknown), a } \\
\text { (proximal, dist }\end{array}$ & $\begin{array}{l}\text { atio. } \\
\text { own), smoking st } \\
\text { ake }(0,0.1-4.9,5 \\
\text { unknown) and }\end{array}$ & $\begin{array}{l}\text { ever, past, current, } \\
, \geqslant 15.0 \mathrm{~g} \mathrm{~d}^{-1} \text { ), ca } \\
\text { diagnosis (continu }\end{array}$ & $\begin{array}{l}\text { vn), body mass in } \\
\text { ge (I-IV or unkn }\end{array}$ & $\begin{array}{l}8.5,18.5-24.9,25 \\
\text { de of tumour dif }\end{array}$ & $\begin{array}{l}30.0-34 \\
\text { ation (we }\end{array}$ \\
\hline
\end{tabular}

whichever came first. Cox proportional hazards regression models were used to calculate hazard ratios (HRs) of death or death as a result of CRC. Test for trend was performed using the median value for each quintile as a continuous variable in the regression models. The Cox models were tested for and met the assumption of proportionality. Survival curves by tertile of dietary insulin scores were generated using the Kaplan-Meier method, and statistical significance was measured using the log-rank test. Tertiles were used instead of quintiles for ease of graphical viewing.

In multivariable analyses, we adjusted for known prognostic factors and potential confounders for CRC survival, including age at diagnosis, sex, cancer stage, grade of tumour differentiation, primary tumour location, year of diagnosis, BMI, physical activity, smoking status, and alcohol intake. We calculated partial Spearman correlation coefficients between dietary insulin scores and relevant biomarkers, adjusted for age at blood collection, sex, BMI, and energy intake. Tests of interaction between dietary insulin scores and potential effect modifiers were assessed by entering in the model the cross product of dietary insulin score as a continuous variable and the stratification variable, evaluated by the likelihood ratio test. All analyses were performed with SAS 9.4 statistical package. All $P$-values were two sided.

\section{RESULTS}

Among 2006 eligible participants with CRC, there were 815 deaths, 343 of which were documented as CRC-specific deaths. The median follow-up period from date of diagnosis for patients who were alive was 12.7 years (range: 2-35.9 years). Baseline characteristics of the 2006 patients are shown in Table 1 by quintile of post-diagnosis dietary insulin score. In general, patients with higher dietary insulin index were older and less likely to smoke, and consumed more carbohydrates and less fat, protein, and alcohol.

Higher post-diagnosis dietary insulin scores were associated with a statistically significant increase in the risk of both CRCspecific and overall mortality (Table 2). Kaplan-Meier curves by dietary insulin score are shown in Figure 1. The pooled adjusted HRs for CRC-specific mortality comparing the highest to the lowest quintiles were 1.82 (95\% CI: $\left.1.20-2.75, P_{\text {trend }}=0.006\right)$ for dietary insulin load and 1.66 (95\% CI: $\left.1.10-2.50, P_{\text {trend }}=0.004\right)$ for dietary insulin index. We also observed an increased risk of overall mortality, with adjusted HRs of 1.33 (95\% CI: 1.03-1.72, $\left.P_{\text {trend }}=0.03\right)$ for dietary insulin load and 1.32 (95\% CI: $1.02-$ $\left.1.71, P_{\text {trend }}=0.02\right)$ for dietary insulin index, comparing extreme quintiles. Though correlated with post-diagnosis dietary insulin scores $(r=0.67$ and 0.55 for dietary insulin load and dietary insulin index, respectively), pre-diagnosis dietary insulin scores were not statistically significantly associated with survival (data not shown). After further adjustment for pre-diagnosis dietary insulin scores, post-diagnosis dietary insulin scores remained statistically significantly associated with survival.

We next evaluated whether the association of dietary insulin scores with CRC-specific mortality could be explained away by other dietary characteristics. Although post-diagnosis dietary glycaemic load and glycaemic index were associated with survival (Supplementary Table 2), inclusion of dietary glycaemic scores into the multivariable model did not change the statistically significant association between dietary insulin scores and CRC-specific mortality (Supplementary Table 3). The association also remained materially unchanged after further adjustment for consumption of red meat, vegetables, and fruits, as well as overall dietary patterns (Supplementary Table 3). Despite the addition of single food items in Supplementary Table 1 into the model both singly and in combination, we continued to observe a statistically significant association between higher dietary insulin scores and worse outcome. 
A
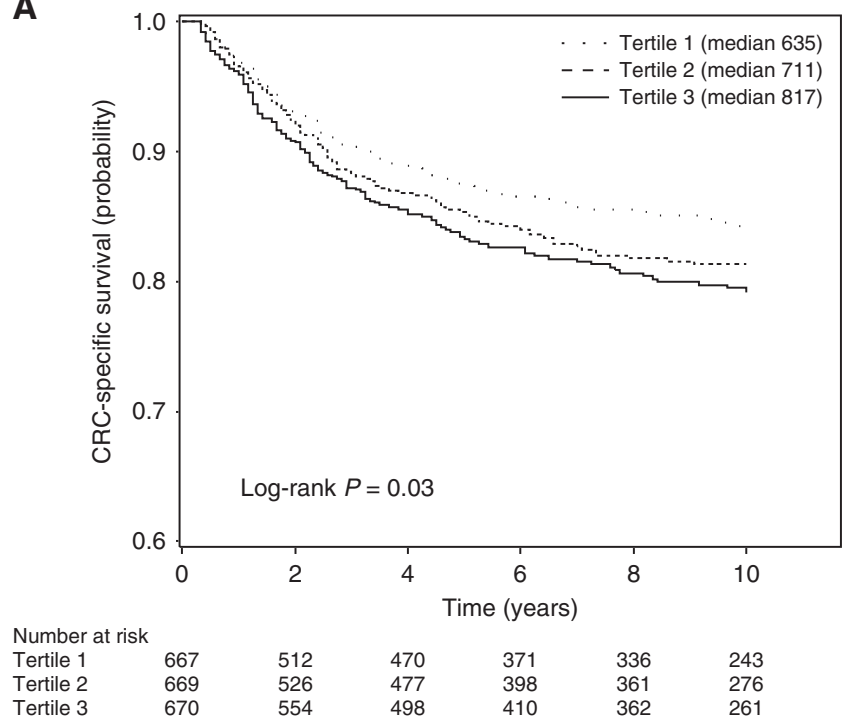

$\begin{array}{llllll}\text { Tertile } 3 & 670 & 554 & 498 & 410 & 362\end{array}$

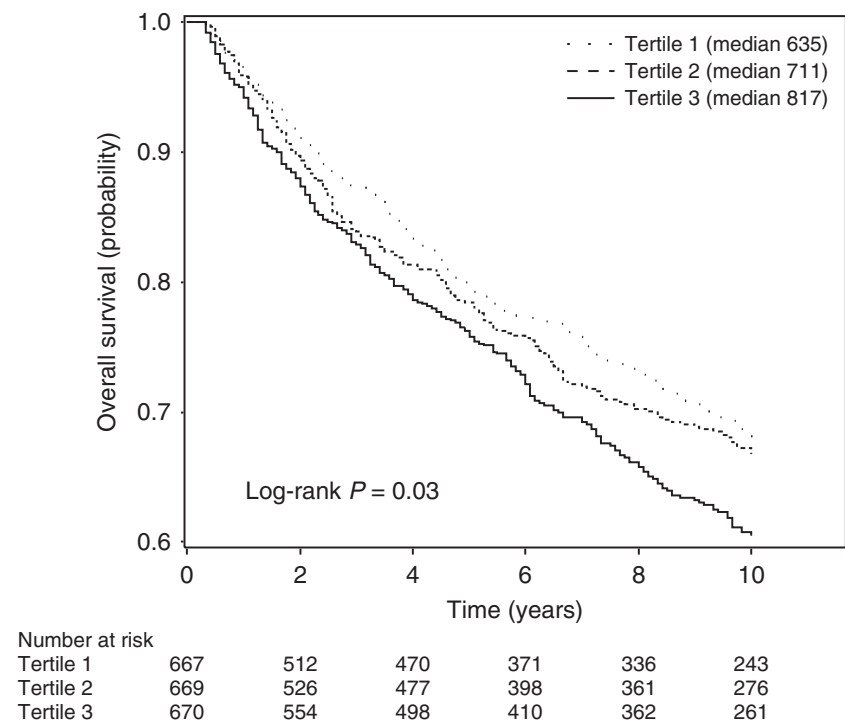

B

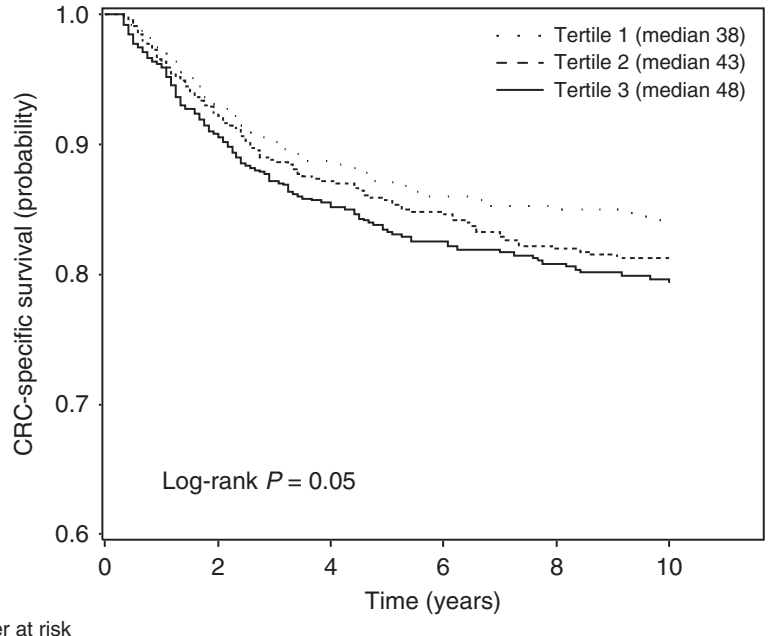

$\begin{array}{lllllll}\text { Number at risk } & & & & & & \\ \text { Tertile 1 } & 668 & 512 & 468 & 368 & 335 & 243 \\ \text { Tertile 2 } & 670 & 527 & 480 & 403 & 365 & 278\end{array}$

Figure 1. Kaplan-Meier curves of colorectal cancer-specific and overall survival among patients with colorectal cancer by tertile of postdiagnosis (A) dietary insulin load or (B) dietary insulin index. Log-rank $P$-values were calculated using extreme tertiles.

To address the possibility that changes in dietary habits could reflect occult recurrence or impending death, we excluded patients who died within 3 months of dietary assessment in our primary analyses. We also conducted sensitivity analyses by extending the exclusion period to 6 months or by excluding patients with stage IV disease, but our results remained statistically significant. To address the possible impact of active treatment on diet, we conducted sensitivity analyses by excluding patients with dietary assessment collected within 9 months after diagnosis, but continued to see a consistent relationship between higher dietary insulin scores and worse survival.

We examined the correlation between dietary insulin scores and relevant biomarkers at the time of blood collection. We observed a negative correlation of dietary insulin scores with adiponectin $(P=0.02$ and 0.05 for dietary insulin load and dietary insulin index, respectively) and no correlation with C-peptide or IGFBP-1.

We also examined the association of dietary insulin index with CRC-specific mortality stratified by predictors of patient outcome (Table 3) and relevant biomarkers (Table 4). The increase in CRCspecific mortality associated with higher dietary insulin index was more apparent among patients with $\mathrm{BMI} \geqslant 25 \mathrm{~kg} \mathrm{~m}^{-2}$ (HR: 2.32; 95\% CI: $1.21-4.46)$ than $\mathrm{BMI}<25 \mathrm{~kg} \mathrm{~m}^{-2}$ (HR: $1.14 ; 95 \% \mathrm{CI}$ : $\left.0.67-1.93 ; P_{\text {interaction }}=0.01\right)$. No statistically significant interactions were seen with age, sex, physical activity, alcohol intake, diagnosis period, time between diagnosis and dietary assessment, cancer stage, grade of tumour differentiation, location of primary tumour, or levels of adiponectin, C-peptide, or IGFBP-1. Dietary insulin load had similar interactions with these covariates (data not shown).

\section{DISCUSSION}

Higher dietary insulin load and dietary insulin index after diagnosis of CRC were associated with increased risk of CRC-specific and overall mortality. Moreover, the increased mortality associated with higher dietary insulin scores was principally observed among patients who were overweight or obese.

Dietary insulin scores have not been extensively studied in relation to development and progression of CRC. In a nested casecontrol study, we observed that higher dietary insulin load or index was not associated with an increase in CRC risk (Bao et al, 2010). 
Table 3. HRs for CRC-specific mortality among patients with colorectal cancer by quintile of post-diagnosis dietary insulin index stratified by predictors of patient outcome

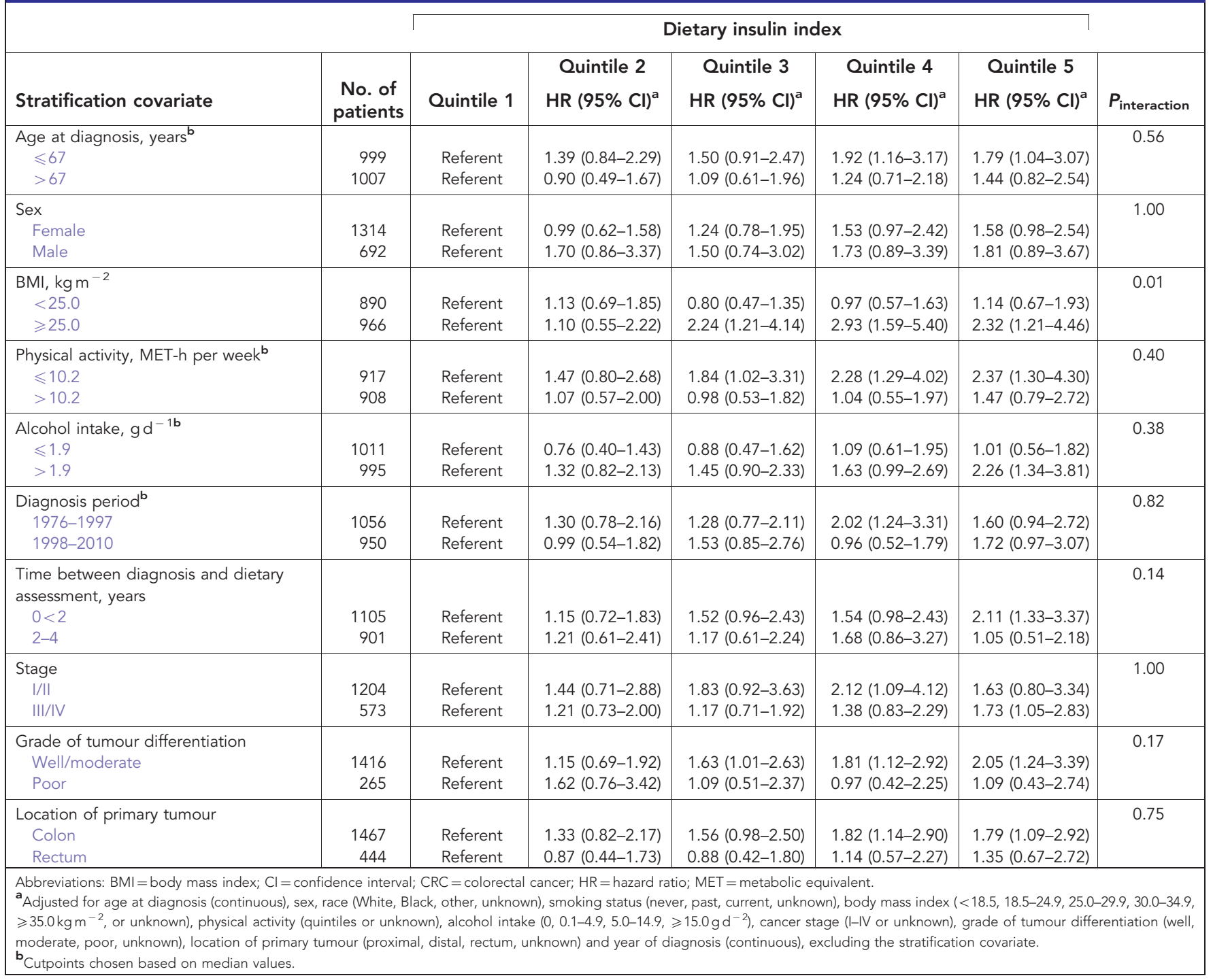

Table 4. HRs for CRC-specific mortality among patients with colorectal cancer by quintile of post-diagnosis dietary insulin index stratified by relevant biomarkers

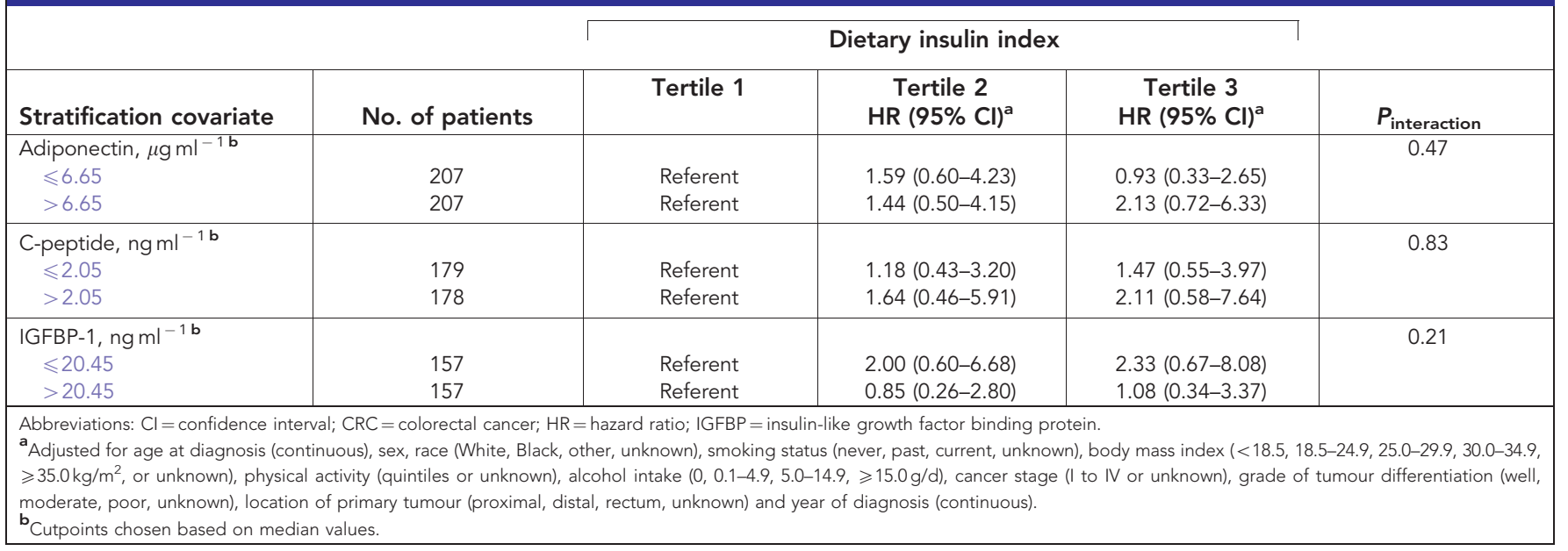


In the current study, pre-diagnosis dietary insulin scores were not associated with CRC survival. Moreover, we did not observe a correlation between pre-diagnosis dietary insulin scores and prediagnosis plasma C-peptide, a more stable biomarker of insulin secretion than plasma insulin. One possible explanation is that the insulinogenic content of a diet as represented by the dietary insulin load or index may not contribute to sustained hyperinsulinemia among healthy participants, which is largely determined by the degree of insulin resistance. In this study, however, dietary insulin load and dietary insulin index were both strongly associated with worse patient outcome, suggesting that tumour progression among CRC patients may be influenced by acute postprandial insulin secretion in response to food intake. Our findings are consistent with a recent study using a subset of this population with measured tumour molecular markers $(N=1160)$, in which the overall HR for CRC-specific mortality was 1.19 (95\% CI: 1.02-1.38) for an increase of one standard deviation in dietary insulin index (Keum et al, 2017).

BMI, physical activity, and diet are established risk factors for several types of cancer, including CRC (Friedenreich and Orenstein, 2002; Giovannucci, 2002; Calle et al, 2003; Johnson and Lund, 2007), and these modifiable behaviours are now increasingly recognised as potentially important risk factors for CRC recurrence, progression, and death (Meyerhardt et al, 2003; Dignam et al, 2006; Haydon et al, 2006; Meyerhardt et al, 2006a, b, 2007). Although the biological mediators of this increased risk of recurrence and death are poorly defined, hyperinsulinemia and perturbations in the insulin-like growth factor axis have been proposed as underlying mechanisms for these observations (Sandhu et al, 2002; Giovannucci, 2003b; Calle and Kaaks, 2004; Davies et al, 2006). This hypothesis is supported by laboratory studies of intestinal epithelial cells and colon cancer cell lines, in which insulin binds to the insulin receptor on the cancer cell surface and stimulates cell growth, while inhibiting apoptosis, suggesting that insulin may act directly as a mitogen for colon cancer cells (Tran et al, 1996; Desbois-Mouthon et al, 2000; Taniguchi et al, 2006; Tran et al, 2006; Shi et al, 2007; Sun and Jin, 2008). Among patients with non-metastatic CRC, elevated levels of plasma C-peptide were associated with an increased risk of CRCspecific mortality (Wolpin et al, 2009). In addition, the association between dietary insulin scores and CRC survival appeared more apparent when CRC is negative for PIK3CA mutation and fatty acid synthase (FASN), two molecular markers linked to the insulin signalling pathway (Keum et al, 2017). A recent study demonstrated that dietary sugar intake increases liver tumour incidence in female mice (Healy et al, 2016), suggesting that a higher dietary insulin index may affect CRC progression by promoting metastasis to the liver.

We noted a greater association of higher dietary insulin scores with increased CRC-specific mortality among overweight or obese patients, suggesting that dietary factors may play a more critical role among those with established insulin resistance. Interestingly, among healthy participants, those with higher dietary insulin scores had increased plasma concentrations of triglycerides (a marker of insulin secretion), and this association was strongest among obese participants (Nimptsch et al, 2011).

The current study has several strengths, including the prospective design, large sample size, long follow-up period, high follow-up rate, and detailed information on other prognostic factors. The procedure for developing the food insulin index was performed under highly standardised conditions (Holt et al, 1997) and shown to be an accurate measure of actual postprandial insulin response evoked by composite meals (Bao et al, 2009). Additionally, dietary insulin scores were correlated with plasma levels of relevant biomarkers, including triglyceride in a healthy population (Nimptsch et al, 2011) and adiponectin in this study, confirming that the estimates of dietary insulin scores are able to predict an expected biological response.

Our work has several limitations. Patients with either occult cancer recurrence or other poor prognostic characteristics may have consumed a high insulinogenic diet as an alternative source of needed calories. To minimise this potential bias, we excluded patients who died within 3 months of dietary assessment. When we extended this restriction to 6 months, we continued to observe a statistically significant association between higher dietary insulin scores and worse survival.

We also considered the possibility that patients who consumed a high insulinogenic diet after cancer diagnosis may have consumed a similar diet before diagnosis and acquired biologically more aggressive tumours. However, we did not observe any statistically significant association between dietary insulin scores and tumourrelated characteristics associated with survival, including stage and grade of tumour differentiation. Furthermore, with the availability of repeated dietary measures in NHS and HPFS, we were able to control for pre-diagnosis dietary insulin scores, with no appreciable change in our results.

Another potential concern with the food insulin index values is that they were derived from lean university students (Holt et al, 1997), whose absolute insulin response may be different from that of the older and heavier individuals; however, the method is valid if the increase in insulin levels induced by a food, that is, the relative insulin response, is comparable between the two groups. Indeed, in the biomarker validation study (Nimptsch et al, 2011), the association observed between dietary insulin index and triglyceride concentrations was strongest among obese participants, indicating that the general method used to develop the insulin index also applies to heavier individuals.

\section{CONCLUSIONS}

In summary, consumption of a dietary pattern characterised by higher dietary insulin scores was statistically significantly associated with increased cancer-specific and overall mortality among CRC patients from two large prospective cohort studies. These data offer further support for the link between energy balance factors and CRC progression and reinforces the need for continued research into the role of these pathways in CRC pathogenesis. Our findings will help to guide dietary recommendations for CRC patients and offer potentially modifiable opportunities to improve patient survival.

\section{ACKNOWLEDGEMENTS}

We would like to thank the participants and staff of NHS and HPFS for their valuable contributions as well as the following state cancer registries for their help: $\mathrm{AL}, \mathrm{AZ}, \mathrm{AR}, \mathrm{CA}, \mathrm{CO}, \mathrm{CT}, \mathrm{DE}, \mathrm{FL}$, GA, ID, IL, IN, IA, KY, LA, ME, MD, MA, MI, NE, NH, NJ, NY, NC, ND, OH, OK, OR, PA, RI, SC, TN, TX, VA, WA, WY. The authors assume full responsibility for analyses and interpretation of these data. NHS is supported by NIH grants UM1 CA186107, P01 CA87969, and R01 CA49449. HPFS is supported by NIH grant UM1 CA167552. Additional support from the National Cancer Institute at the National Institutes of Health (K24 DK098311 to ATC; R01 CA137178 to ATC; P50 CA127003 to CSF; K07 CA148894 to KN), from the Damon Runyon Cancer Research Foundation (to ATC), from the American Institute for Cancer Research (to KW), from the American Society of Clinical Oncology (ASCO) Career Development Award (to KN), and from the Entertainment Industry Foundation's National Colorectal Cancer Research Alliance (NCCRA). 


\section{CONFLICT OF INTEREST}

The authors declare no conflict of interest.

\section{REFERENCES}

Bao J, de Jong V, Atkinson F, Petocz P, Brand-Miller JC (2009) Food insulin index: physiologic basis for predicting insulin demand evoked by composite meals. Am J Clin Nutr 90(4): 986-992.

Bao Y, Nimptsch K, Meyerhardt JA, Chan AT, Ng K, Michaud DS, Brand-Miller JC, Willett WC, Giovannucci E, Fuchs CS (2010) Dietary insulin load, dietary insulin index, and colorectal cancer. Cancer Epidemiol Biomarkers Prev 19(12): 3020-3026.

Calle EE, Kaaks R (2004) Overweight, obesity and cancer: epidemiological evidence and proposed mechanisms. Nat Rev Cancer 4(8): 579-591.

Calle EE, Rodriguez C, Walker-Thurmond K, Thun MJ (2003) Overweight, obesity, and mortality from cancer in a prospectively studied cohort of U.S. adults. N Engl J Med 348(17): 1625-1638.

Chong DQ, Mehta RS, Song M, Kedrin D, Meyerhardt JA, Ng K, Wu K, Fuchs CS, Giovannucci EL, Ogino S, Chan AT (2015) Prediagnostic plasma adiponectin and survival among patients with colorectal cancer. Cancer Prev Res (Phila) 8(12): 1138-1145.

Colditz GA, Manson JE, Hankinson SE (1997) The Nurses' Health Study: 20-year contribution to the understanding of health among women. $J$ Womens Health 6(1): 49-62.

Davies M, Gupta S, Goldspink G, Winslet M (2006) The insulin-like growth factor system and colorectal cancer: clinical and experimental evidence. Int J Colorectal Dis 21(3): 201-208.

Desbois-Mouthon C, Cadoret A, Blivet-Van Eggelpoel MJ, Bertrand F, Caron M, Atfi A, Cherqui G, Capeau J (2000) Insulin-mediated cell proliferation and survival involve inhibition of c-"Jun $\mathrm{N}$-"terminal kinases through a phosphatidylinositol 3-kinase- and mitogen-activated protein kinase phosphatase-1-dependent pathway. Endocrinology 141(3): 922-931.

Dignam JJ, Polite BN, Yothers G, Raich P, Colangelo L, O'Connell MJ, Wolmark N (2006) Body mass index and outcomes in patients who receive adjuvant chemotherapy for colon cancer. J Natl Cancer Inst 98(22): $1647-1654$.

Feskanich D, Rimm EB, Giovannucci EL, Colditz GA, Stampfer MJ, Litin LB, Willett WC (1993) Reproducibility and validity of food intake measurements from a semiquantitative food frequency questionnaire. J Am Diet Assoc 93(7): 790-796.

Friedenreich CM, Orenstein MR (2002) Physical activity and cancer prevention: etiologic evidence and biological mechanisms. J Nutr 132(11 Suppl): 3456S-3464S.

Giovannucci E (2002) Modifiable risk factors for colon cancer. Gastroenterol Clin North Am 31(4): 925-943.

Giovannucci E (2003a) Diet, body weight, and colorectal cancer: a summary of the epidemiologic evidence. J Womens Health (Larchmt) 12(2): 173-182.

Giovannucci E (2003b) Nutrition, insulin, insulin-like growth factors and cancer. Horm Metab Res 35(11-12): 694-704.

Giovannucci E, Colditz GA, Stampfer MJ, Hunter D, Rosner BA, Willett WC, Speizer FE (1994a) A prospective study of cigarette smoking and risk of colorectal adenoma and colorectal cancer in U.S. women. J Natl Cancer Inst 86(3): 192-199.

Giovannucci E, Rimm EB, Stampfer MJ, Colditz GA, Ascherio A, Kearney J, Willett WC (1994b) A prospective study of cigarette smoking and risk of colorectal adenoma and colorectal cancer in U.S. men. J Natl Cancer Inst 86(3): 183-191.

Haydon AM, Macinnis RJ, English DR, Giles GG (2006) Effect of physical activity and body size on survival after diagnosis with colorectal cancer. Gut 55(1): 62-67.

Healy ME, Lahiri S, Hargett SR, Chow JD, Byrne FL, Breen DS, Kenwood BM, Taddeo EP, Lackner C, Caldwell SH, Hoehn KL (2016) Dietary sugar intake increases liver tumor incidence in female mice. Sci Rep 6: 22292.

Holt SH, Miller JC, Petocz P (1997) An insulin index of foods: the insulin demand generated by 1000-kJ portions of common foods. Am J Clin Nutr 66(5): 1264-1276.

Johnson IT, Lund EK (2007) Review article: nutrition, obesity and colorectal cancer. Aliment Pharmacol Ther 26(2): 161-181.

Kaaks R, Toniolo P, Akhmedkhanov A, Lukanova A, Biessy C, Dechaud H, Rinaldi S, Zeleniuch-Jacquotte A, Shore RE, Riboli E (2000) Serum
C-"peptide, insulin-like growth factor (IGF)-I, IGF-binding proteins, and colorectal cancer risk in women. J Natl Cancer Inst 92(19): $1592-1600$.

Keum N, Yuan C, Nishihara R, Zoltick E, Hamada T, Martinez Fernandez A, Zhang X, Hanyuda A, Liu L, Kosumi K, Nowak JA, Jhun I, Soong TR, Morikawa T, Tabung FK, Qian ZR, Fuchs CS, Meyerhardt JA, Chan AT, Ng K, Ogino S, Giovannucci EL, Wu K (2017) Dietary glycemic and insulin scores and colorectal cancer survival by tumor molecular biomarkers. Int J Cancer 140(12): 2648-2656.

Larsson SC, Orsini N, Wolk A (2005) Diabetes mellitus and risk of colorectal cancer: a meta-analysis. J Natl Cancer Inst 97(22): 1679-1687.

Ma J, Giovannucci E, Pollak M, Leavitt A, Tao Y, Gaziano JM, Stampfer MJ (2004) A prospective study of plasma C-"peptide and colorectal cancer risk in men. J Natl Cancer Inst 96(7): 546-553.

Meyerhardt JA, Catalano PJ, Haller DG, Mayer RJ, Benson 3rd AB, Macdonald JS, Fuchs CS (2003) Influence of body mass index on outcomes and treatment-related toxicity in patients with colon carcinoma. Cancer 98(3): 484-495.

Meyerhardt JA, Giovannucci EL, Holmes MD, Chan AT, Chan JA, Colditz GA, Fuchs CS (2006a) Physical activity and survival after colorectal cancer diagnosis. J Clin Oncol 24(22): 3527-3534.

Meyerhardt JA, Heseltine D, Niedzwiecki D, Hollis D, Saltz LB, Mayer RJ, Thomas J, Nelson H, Whittom R, Hantel A, Schilsky RL, Fuchs CS (2006b) Impact of physical activity on cancer recurrence and survival in patients with stage III colon cancer: findings from CALGB 89803. J Clin Oncol 24(22): 3535-3541.

Meyerhardt JA, Niedzwiecki D, Hollis D, Saltz LB, Hu FB, Mayer RJ, Nelson H, Whittom R, Hantel A, Thomas J, Fuchs CS (2007) Association of dietary patterns with cancer recurrence and survival in patients with stage III colon cancer. Jama 298(7): 754-764.

Meyerhardt JA, Sato K, Niedzwiecki D, Ye C, Saltz LB, Mayer RJ, Mowat RB, Whittom R, Hantel A, Benson A, Wigler DS, Venook A, Fuchs CS (2012) Dietary glycemic load and cancer recurrence and survival in patients with stage III colon cancer: findings from CALGB 89803. J Natl Cancer Inst 104(22): 1702-1711.

Moghaddam AA, Woodward M, Huxley R (2007) Obesity and risk of colorectal cancer: a meta-analysis of 31 studies with 70000 events. Cancer Epidemiol Biomarkers Prev 16(12): 2533-2547.

Nimptsch K, Brand-Miller JC, Franz M, Sampson L, Willett WC, Giovannucci E (2011) Dietary insulin index and insulin load in relation to biomarkers of glycemic control, plasma lipids, and inflammation markers. Am J Clin Nutr 94(1): 182-190.

Rimm EB, Giovannucci EL, Willett WC, Colditz GA, Ascherio A, Rosner B, Stampfer MJ (1991) Prospective study of alcohol consumption and risk of coronary disease in men. Lancet 338(8765): 464-468.

Salvini S, Hunter DJ, Sampson L, Stampfer MJ, Colditz GA, Rosner B, Willett WC (1989) Food-based validation of a dietary questionnaire: the effects of week-to-week variation in food consumption. Int J Epidemiol 18(4): 858-867.

Samad AK, Taylor RS, Marshall T, Chapman MA (2005) A meta-analysis of the association of physical activity with reduced risk of colorectal cancer. Colorectal Dis 7(3): 204-213.

Sandhu MS, Dunger DB, Giovannucci EL (2002) Insulin, insulin-like growth factor-I (IGF-I), IGF binding proteins, their biologic interactions, and colorectal cancer. J Natl Cancer Inst 94(13): 972-980.

Sathiakumar N, Delzell E, Abdalla O (1998) Using the National Death Index to obtain underlying cause of death codes. J Occup Env Med 40(9): 808-813.

Schoen RE, Tangen CM, Kuller LH, Burke GL, Cushman M, Tracy RP, Dobs A, Savage PJ (1999) Increased blood glucose and insulin, body size, and incident colorectal cancer. J Natl Cancer Inst 91(13): $1147-1154$.

Shi B, Sepp-Lorenzino L, Prisco M, Linsley P, deAngelis T, Baserga R (2007) Micro RNA 145 targets the insulin receptor substrate- 1 and inhibits the growth of colon cancer cells. J Biol Chem 282(45): 32582-32590.

Stampfer MJ, Willett WC, Speizer FE, Dysert DC, Lipnick R, Rosner B, Hennekens CH (1984) Test of the National Death Index. Am J Epidemiol 119(5): 837-839.

Sun J, Jin T (2008) Both Wnt and mTOR signaling pathways are involved in insulin-stimulated proto-oncogene expression in intestinal cells. Cell Signal 20(1): 219-229.

Taniguchi CM, Tran TT, Kondo T, Luo J, Ueki K, Cantley LC, Kahn CR (2006) Phosphoinositide 3-kinase regulatory subunit p85alpha suppresses 
insulin action via positive regulation of PTEN. Proc Natl Acad Sci USA 103(32): 12093-12097.

Tran TT, Medline A, Bruce WR (1996) Insulin promotion of colon tumors in rats. Cancer Epidemiol Biomarkers Prev 5(12): 1013-1015.

Tran TT, Naigamwalla D, Oprescu AI, Lam L, McKeown-Eyssen G, Bruce WR, Giacca A (2006) Hyperinsulinemia, but not other factors associated with insulin resistance, acutely enhances colorectal epithelial proliferation in'vivo. Endocrinology 147(4): 1830-1837.

Wei EK, Ma J, Pollak MN, Rifai N, Fuchs CS, Hankinson SE, Giovannucci E (2005) A prospective study of C-"peptide, insulin-like growth factor-I, insulin-like growth factor binding protein-1, and the risk of colorectal cancer in women. Cancer Epidemiol Biomarkers Prev 14(4): 850-855.
Willett W, Stampfer MJ (1986) Total energy intake: implications for epidemiologic analyses. Am J Epidemiol 124(1): 17-27.

Wolpin BM, Meyerhardt JA, Chan AT, Ng K, Chan JA, Wu K, Pollak MN, Giovannucci EL, Fuchs CS (2009) Insulin, the insulin-like growth factor axis, and mortality in patients with nonmetastatic colorectal cancer. J Clin Oncol 27(2): 176-185.

This work is published under the standard license to publish agreement. After 12 months the work will become freely available and the license terms will switch to a Creative Commons AttributionNonCommercial-Share Alike 4.0 Unported License.

Supplementary Information accompanies this paper on British Journal of Cancer website (http://www.nature.com/bjc) 